\title{
Why is autophagy important in human diseases?
}

\author{
Kaipeng Jing ${ }^{1,3}$ and Kyu Lim ${ }^{1,2,3,4}$ \\ ${ }^{1}$ Department of Biochemistry \\ College of Medicine \\ ${ }^{2}$ Cancer Research Institute and \\ ${ }^{3}$ Infection Signaling Network Research Center \\ Chungnam National University \\ Daejeon 301-747, Korea \\ ${ }^{4}$ Corresponding author: Tel, 82-42-580-8223; \\ Fax, 82-42-580-8121; E-mail, kyulim@ @nu.ac.kr \\ http://dx.doi.org/10.3858/emm.2012.44.2.028
}

Accepted 8 December 2011

Available Online 19 January 2012

Abbreviations: ATG, autophagy-related; LC3, microtubule-associated protein 1 light chain 3; UPS, ubiquitin-proteasome system

\begin{abstract}
The process of macroautophagy (referred to hereafter as autophagy), is generally characterized by the prominent formation of autophagic vesicles in the cytoplasm. In the past decades, studies of autophagy have been vastly expanded. As an essential process to maintain cellular homeostasis and functions, autophagy is responsible for the lysosome-mediated degradation of damaged proteins and organelles, and thus misregulation of autophagy can result in a variety of pathological conditions in human beings. Although our understanding of regulatory pathways that control autophagy is still limited, an increasing number of studies have shed light on the importance of autophagy in a wide range of physiological processes and human diseases. The goal of the reviews in the current issue is to provide a general overview of current knowledge on autophagy. The machinery and regulation of autophagy were outlined with special attention to its role in diabetes, neurodegenerative disorders, infectious diseases and cancer.
\end{abstract}

Keywords: autophagy; disease; physiology

\section{What is autophagy?}

Macroautophagy (referred to hereafter as autophagy) is a genetically regulated and dynamic process associated with the formation of autophagosome, a double-membrane cytoplasmic vesicle that engulfs cellular components. The autophagosome formation starts at phagophore (also known as isolation membrane or sequestering membrane) and requires the conjugation of microtubule-associated protein 1 light chain 3 (LC3), which regulates the phagophore expansion and completion of the sequestering vesicle (Xie et al., 2008). The completed autophagosome then fuse with lysosome, becoming autolysosome. Sequestered components are degraded by lysosomal hydrolases and released into the cytosol by lysosomal efflux permeases. This lysosome-mediated degradation system plays a regulatory role in mammalian cell biology by clearing damaged organelles and recycling autophagy-derived nutrients (Figure 1).

\section{General functions of autophagy}

The process of protein degradation and organelle turnover is required for the survival of cells, and the disruption of this process can result in the abnormal cell growth or cell death, leading to various disease states (Klionsky and Emr, 2000). In eukaryotic cells, this process is mainly regulated by two major systems named the ubiquitin-proteasome system (UPS) and autophagy, which work cooperatively to control intracellular protein degradation and organelle turnover (Klionsky and Emr, 2000; Rubinsztein, 2006). Whereas the UPS specializes in short-lived protein degradation, autophagy mainly functions to degrade long-lived proteins and damaged organelles (Rubinsztein, 2006; Nedelsky et al., 2008). Although autophagy is considered to be a nonselective degradation process of engulfment of cytoplasm traditionally, increasing evidence indicates the existence of selective autophagy which specifically degrades mitochondria (mitophagy), endoplasmic reticulum (reticulophagy), microorganisms (xenophagy) and aggregated proteins (aggrephagy), etc. (Johansen and Lamark, 2011).

Depending on the different cellular contexts and stimuli, the outcome of autophagy can promote either 


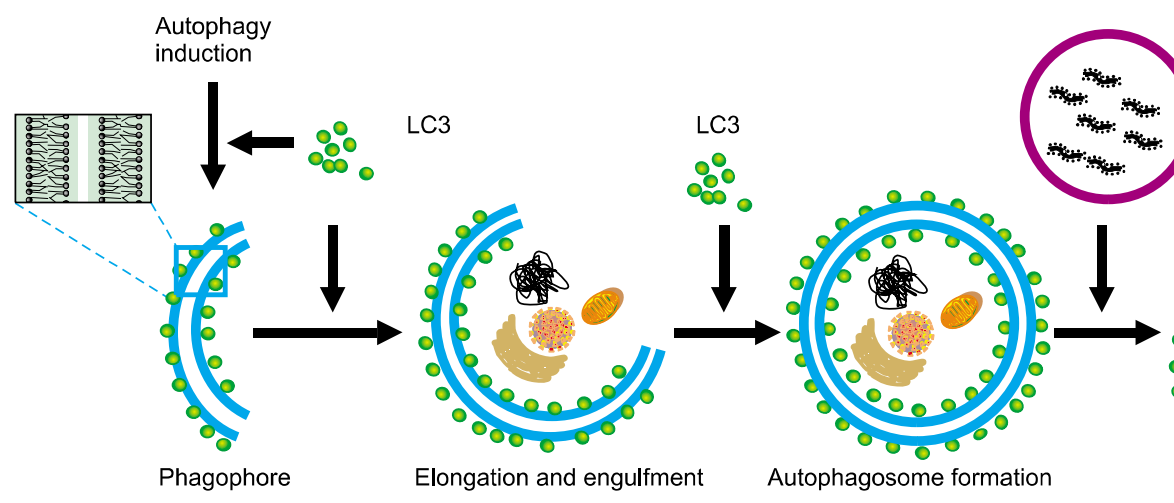

Lysosome
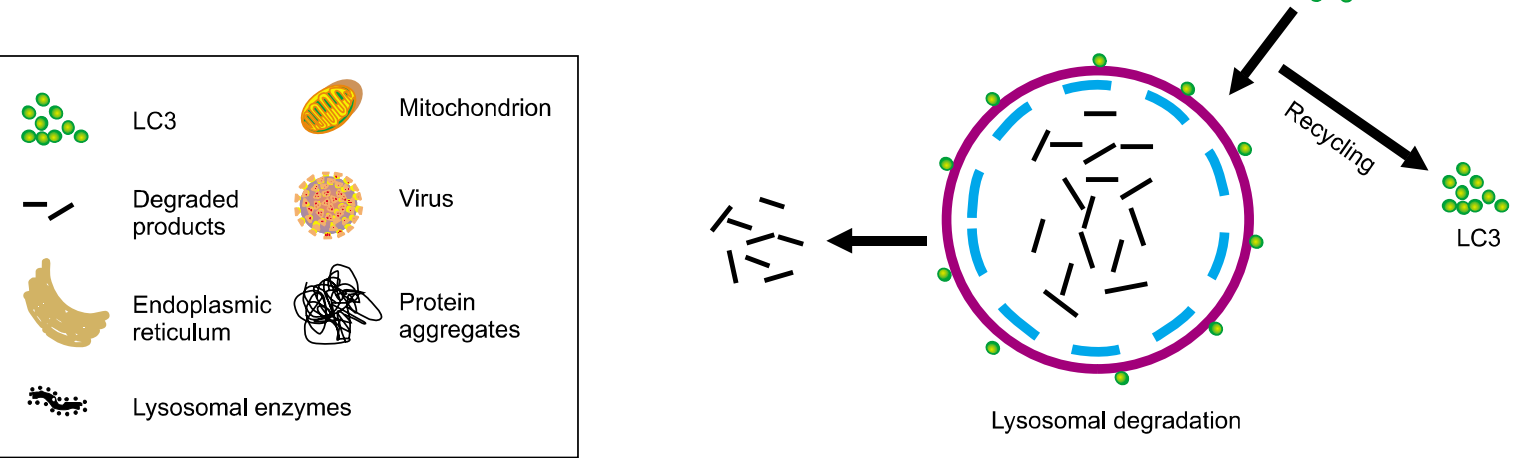

Lysosomal degradation

Figure 1. Schematic diagram of autophagic progression. Autophagy induction signal leads to form a sequestering membrane called phagophore. Following a sequence of ubiquitination-like reactions, LC3 conjugates to the sequestering membrane and controls the elongation of phagophore. As the phagophore expands, cytoplasmic constituents, including organelles such as mitochondria and endoplasmic reticulum, aggregated proteins and foreign organisms (bacteria and virus) are enwrapped. At the end of elongation, sequestering membrane closes and results in the formation of a double-membrane vesicle, autophagosome. Once the autophagosome is formed, it is delivered to fuse with lysosome to form autolysosome for degradation. Lysosomal hydrolases degrades the cargo together with the inner membrane of autophagosome, and LC3 from the outer membraneas well as the autophagy-derived nutrients are recycled. This autophagic process can act as a mechanism to keep homeostatic balance and support cell survival. However, it can also cause cell death directly or indirectly.

cell survival or cell death. However, the mechanisms underlying the dual role for autophagy in deciding the destiny of cells remain unclear. On one hand, since autophagy provides cells with nutrients and eliminates damaged organelles, it is primarily believed to function as a protective mechanism for cell survival, particularly under unfavorable conditions. Indeed, cytotoxic drugs often trigger autophagy activation, and then autophagy inhibition has been reported to potentiate apoptotic cell death induced by several anticancer drugs (Amaravadi et al., 2007; Song et al., 2008). Nevertheless, autophagy impairment can also block or delay the development of cell death (Berry and Baehrecke, 2007; Yuk et al., 2010; Jing et al., 2011), and in some instances, autophagy itself is capable of inducing cell death (Shao et al., 2004; Yousefi et al., 2006). This role of autophagy in enhancing cell death may be related to the degradation of essential factors for cell survival (Yu et al., 2006) or its effect on energy maintenance considering that ATP is required during programmed cell death, such as apoptosis (Qu et al., 2007).

\section{The impacts of autophagy on human physiology and diseases}

Nearly all eukaryotic cells undergo autophagy at a basal level under normal physiological conditions, and cells deficient in autophagy show diffuse abnormal protein accumulation and mitochondria disorganization (Hara et al., 2006; Ebato et al., 2008), suggesting that cells use autophagy to maintain cellular homeostasis by eliminating damaged organelles and protein aggregates, which resist degradation via UPS in normal growing conditions. In addition to the effect on cellular homeostasis maintenance, autophagy also regulates rapid cellular changes essential for mammalian development and differentiation. For instance, it has been demonstrated that autophagy is required in mitochondrial elimination during erythrocyte and adipocyte maturation (Mortensen et al., 2010; Goldman et al., 2011). Furthermore, autophagy is not only responsible for the clearance of abnormal proteins and organelles, but also participates in the removing of infectious agents, including bacteria and viruses from host cells. Recent data from stud- 


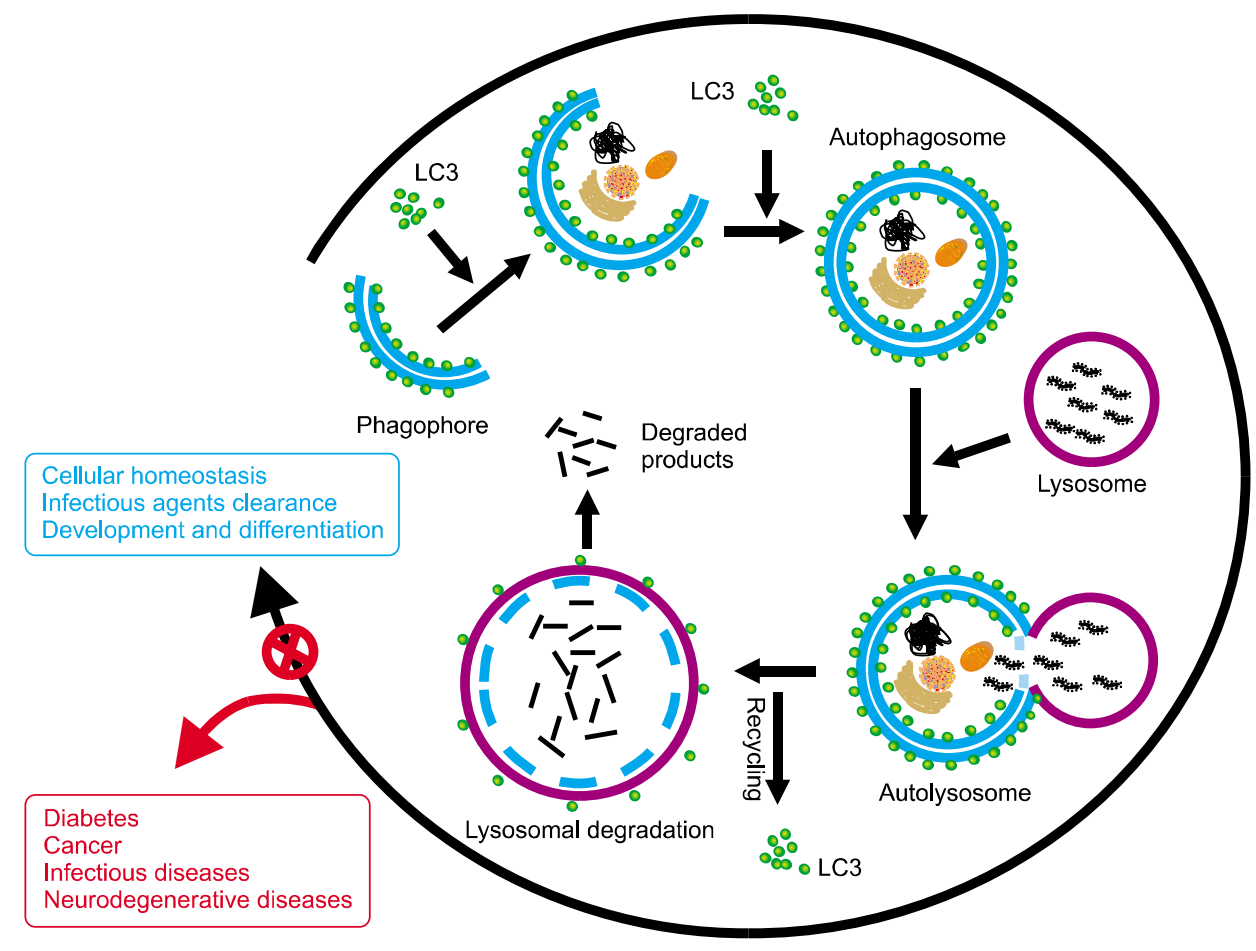

Figure 2. Dysregulated autophagy is involved in several human diseases. Autophagy contributes to maintain cellular homeostasis and is critical in a wide range of normal human physiological processes. Accordingly, a growing number of diseases are linked to the misregulation of autophagic process. $\circledast$, dysregulation of autophagy. ies of cell models show that autophagy upregulation might be valuable for eliminating Mycobacterium tuberculosis (Gutierrez et al., 2004), Streptococcus (Nakagawa et al., 2004), mycobacteria (Singh et al., 2006; Shin et al., 2010) and herpes simplex virus (Talloczy et al., 2006). This function of autophagy appears to be accounted for by both innate and adaptive immune responses (Levine and Deretic, 2007).

Because of the importance of autophagy in mammalian physiology, it is therefore reasonable to assume that autophagy impairment could contribute to human diseases. However, although autophagy was first described morphologically in mammalian cells in 1960s (Deter et al., 1967), until the discovery of a group of autophagy-related (ATG) genes in 1990s (Tsukada and Ohsumi, 1993), the role of autophagy in various human disease states was unclear, and only in the past decade has the connection between autophagy and human disease become the subject of intense study. To date, autophagy has been linked to a growing list of diseases, and it appears that more autophagy-associated diseases will be discovered in the future. Although the exact mechanisms underlying the autophagy-associated diseases at the molecular level remain not fully understood, the recent advances in autophagy research present a potential target for manipulation of autophagy in human diseases (Figure 2).

The following reviews aim at outlining our current understanding of mechanisms involved in autophagy activation, and its important roles in human physiology and diseases, including diabetes mellitus, neurodegenerative disorders, infectious diseases and cancer.

\section{Acknowledgements}

This work was supported by the National Research Foundation of Korea (NRF) grant funded by the Ministry of Education, Science and Technology (2011-0006232, 20110013263 and 2011-0003060).

\section{References}

Amaravadi RK, Yu D, Lum JJ, Bui T, Christophorou MA, Evan GI, Thomas-Tikhonenko A, Thompson CB. Autophagy inhibition enhances therapy-induced apoptosis in a Mycinduced model of lymphoma. J Clin Invest 2007;117:326-36

Berry DL, Baehrecke EH. Growth arrest and autophagy are required for salivary gland cell degradation in Drosophila. Cell 2007;131:1137-48

Deter RL, Baudhuin P, De Duve C. Participation of lysosomes in cellular autophagy induced in rat liver by glucagon. J Cell Biol 1967;35:C11-6

Ebato C, Uchida T, Arakawa M, Komatsu M, Ueno T, Komiya K, Azuma K, Hirose T, Tanaka K, Kominami E, Kawamori R, Fujitani $\mathrm{Y}$, Watada H. Autophagy is important in islet homeostasis and compensatory increase of beta cell mass in response to high-fat diet. Cell Metab 2008;8:325-32 
Goldman SJ, Zhang Y, Jin S. Autophagic degradation of mitochondria in white adipose tissue differentiation. Antioxid Redox Signal 2011;14:1971-8

Gutierrez MG, Master SS, Singh SB, Taylor GA, Colombo MI, Deretic V. Autophagy is a defense mechanism inhibiting BCG and Mycobacterium tuberculosis survival in infected macrophages. Cell 2004;119:753-66

Hara T, Nakamura K, Matsui M, Yamamoto A, Nakahara Y, Suzuki-Migishima R, Yokoyama M, Mishima K, Saito I, Okano H, Mizushima N. Suppression of basal autophagy in neural cells causes neurodegenerative disease in mice. Nature 2006;441:885-9

Jing K, Song KS, Shin S, Kim N, Jeong S, Oh HR, Park JH, Seo KS, Heo JY, Han J, Park JI, Han C, Wu T, Kweon GR, Park SK, Yoon WH, Hwang BD, Lim K. Docosahexaenoic acid induces autophagy through p53/AMPK/mTOR signaling and promotes apoptosis in human cancer cells harboring wild-type p53. Autophagy 2011;7:1348-58

Johansen T, Lamark T. Selective autophagy mediated by autophagic adapter proteins. Autophagy 2011;7:279-96

Klionsky DJ, Emr SD. Autophagy as a regulated pathway of cellular degradation. Science 2000;290:1717-21

Levine B, Deretic V. Unveiling the roles of autophagy in innate and adaptive immunity. Nat Rev Immunol 2007;7:767-77

Mortensen M, Ferguson DJ, Edelmann M, Kessler B, Morten KJ, Komatsu M, Simon AK. Loss of autophagy in erythroid cells leads to defective removal of mitochondria and severe anemia in vivo. Proc Natl Acad Sci USA 2010;107:832-7

Nakagawa I, Amano A, Mizushima N, Yamamoto A, Yamaguchi H, Kamimoto T, Nara A, Funao J, Nakata M, Tsuda K, Hamada S, Yoshimori T. Autophagy defends cells against invading group A Streptococcus. Science 2004; 306:1037-40

Nedelsky NB, Todd PK, Taylor JP. Autophagy and the ubiquitin-proteasome system: collaborators in neuroprotection. Biochim Biophys Acta 2008;1782:691-9

Qu X, Zou Z, Sun Q, Luby-Phelps K, Cheng P, Hogan RN, Gilpin C, Levine B. Autophagy gene-dependent clearance of apoptotic cells during embryonic development. Cell 2007;128:931-46
Rubinsztein DC. The roles of intracellular protein-degradation pathways in neurodegeneration. Nature 2006;443:780-6

Shao Y, Gao Z, Marks PA, Jiang X. Apoptotic and autophagic cell death induced by histone deacetylase inhibitors. Proc Natl Acad Sci USA 2004;101:18030-5

Shin DM, Yuk JM, Lee HM, Lee SH, Son JW, Harding CV, Kim JM, Modlin RL, Jo EK. Mycobacterial lipoprotein activates autophagy via TLR2/1/CD14 and a functional vitamin D receptor signalling. Cell Microbiol 2010;12:1648-65

Singh SB, Davis AS, Taylor GA, Deretic V. Human IRGM induces autophagy to eliminate intracellular mycobacteria. Science 2006;313:1438-41

Song KS, Kim JS, Yun EJ, Kim YR, Seo KS, Park JH, Jung YJ, Park JI, Kweon GR, Yoon WH, Lim K, Hwang BD. Rottlerin induces autophagy and apoptotic cell death through a PKC-delta-independent pathway in HT1080 human fibrosarcoma cells: the protective role of autophagy in apoptosis. Autophagy 2008;4:650-8

Talloczy Z, Virgin HW 4th, Levine B. PKR-dependent autophagic degradation of herpes simplex virus type 1 . Autophagy 2006;2:24-9

Tsukada M, Ohsumi Y. Isolation and characterization of autophagy-defective mutants of Saccharomyces cerevisiae. FEBS Lett 1993;333:169-74

Xie Z, Nair U, Klionsky DJ. Atg8 controls phagophore expansion during autophagosome formation. Mol Biol Cell 2008;19:3290-8

Yousefi S, Perozzo R, Schmid I, Ziemiecki A, Schaffner T, Scapozza L, Brunner T, Simon HU. Calpain-mediated cleavage of Atg5 switches autophagy to apoptosis. Nat Cell Biol 2006;8:1124-32

Yu L, Wan F, Dutta S, Welsh S, Liu Z, Freundt E, Baehrecke $E H$, Lenardo M. Autophagic programmed cell death by selective catalase degradation. Proc Natl Acad Sci USA 2006;103:4952-7

Yuk JM, Shin DM, Song KS, Lim K, Kim KH, Lee SH, Kim JM, Lee JS, Paik TH, Kim JS, Jo EK. Bacillus calmette-guerin cell wall cytoskeleton enhances colon cancer radiosensitivity through autophagy. Autophagy 2010;6:46-60 